\title{
Martial arts education sociology analysis of male gender roles
}

\author{
Kun Liu ${ }^{1, a}$, Na Liüb
}

${ }^{1} \mathrm{PE}$ Department of Jiangxi University of Traditional Chinese Medicine, Nanchang 330004, China

aliuk0791@163.com, b329022334@qq.com

Corresponding author: Na Liu

Keywords: Male temperament, Weakening, Martial arts, neutralization

\begin{abstract}
The Chinese masculinity weakened become a hot topic. Currently, youth groups, especially in the student groups, men and women seem to become a trend, in essence, this kind of phenomenon has constituted the concerns of the characteristics of civil society sex differentiation. This article from the perspective of sociology, cultural science, logic and so on, the contemporary student groups, analyzes the phenomenon of masculinity weakened points lead to masculinity weakened by the following factors: men and women of kindergarten to university teachers' imbalance, in the school sports "masculine" project of erosion, the fashion of misleading, contemporary family roles change of mass media and the change of the mode of production to promote social gender division of labor of the five factors lead to weakening masculinity, and put forward the way to strengthen the martial arts education this to strengthen masculinity.
\end{abstract}

\section{Introduction}

Masculinities from sex role theory, it is the male gender traits or characteristics, is the male has universality, the sex of the essential attributes. But in recent years, China's lack of masculine phenomenon is serious, it may have the pressure of the male adolescents, mood, behavior and psychological produced by negative factors, etc. This article from the sociological Angle of view to elaborate the specific factors of lack of male gender roles.

\section{The lack of male gender roles: a social $q$ is unable to avoid}

Gender role is the individual in the process of social life in accordance with the process of gender norms by yourself. Gender roles for growth plays an important role for personal health. However, in today's society, especially the lack of male gender roles in school education, is not conducive to the all-round development of human beings. From the perspective of social development, men and women is the smallest unit of individuals constitute the society, in the entire social structure, as a man and a woman means to play for a certain sex of a whole set of expectations, that is the sex role."Yin flourishes while Yang declines, however, the current situation seems to be no big change. The male culture erosion happens in the only female, also for a contemporary Chinese society needs systematic problem."

\section{Sociological factors analysis of a lack of gender roles}

We everyone in different social status, the changes of the social roles lead to lack of gender roles, namely man temperament. Social status, it refers to any has from the point of view of social rules the rights and obligations of the social position, whether the position is to is low. Social status is a person in the whole social relations system the location of his own, no matter this position is a natural or social division of labor need to form.S

Men and women from kindergarten to university teachers imbalance - social role changes of structure factors.

Every one of us want to be conform to the requirements of the social members of society, the most important factor is through the contact and interaction with others, learn and internalize social norms, and become a part of the personality, this is man's socialization process. History of law 
(P.I.R ose) think that socialization is the learning of social and cultural beliefs, values, norms and social roles of process. Contact from us, therefore, people born after the family has been to the school to work into the society, this is all part of the social.

Especially in kindergarten, it is the first key battleground of socialization of children. Kindergarten teachers are every child out of the family towards society met first "outsiders", the "outsiders" have a certain "authority", he told the young children's mental and physical growth and development impact is unmatched by parents. However, because of from kindergarten to primary school, the female teacher ratio is high, so the teacher is also a children's access to the most. Female teacher's role and the mother role is very close, and even can be said to be equivalent to a mother's role in the field of school, so they possess relative advantage when it comes to managing their children. But social problems are increasingly prominent. Some children, especially boys from kindergarten to primary school this for decades in the socialization process, has been immersed in the female environment, surrounded by all the time the infected women's temperament, masculinity, seldom influence of man. Small schools in the proportion of male role led to most of the kids due to lack of masculine man, terrible male characteristics of osmosis, then creates a masculine weakening.

The loss of 2.2 in the school sports "masculine" project - social roles change education problems.

Since reform and opening up in 1978, many western sports thought was introduced into our country school sports, school sports development in China has played a huge role in promoting. However, the eastern and western regional differences exist not only, also there are cultural differences, there are more of differences." Any things there are always two sides to everything," some western sports thought was introduced into our country school sports at the same time, also for our country school sports reform has brought some "side effect". Sports "is usually a" happy ", "sport". We have ignored in school physical education "physical education" and sing "happy sports," quality-oriented education, such as "perfect education", but the reform of school physical education for nearly 30 years our students' physique in some important index falling again and again.

Have some scholars think that western sports thoughts "too much emphasis on the psychological and social adaptation, the operational layer and in the practice, ignored the biological concept of sports physical changes to the student, the influence of this effect has a certain relationship with current students physical deterioration". Physical decline, thus become one of the causes of part of the sports injury accidents." School sports injury accident happens to the school and the teacher is too strict imputation prompted some negative and extreme aversion measures arises at the historic moment, thus to avoid some risky projects, hurdles, discus, javelin, gymnastics, long runs, highly confrontational projects are out of sports curriculum view, instead, some relatively" soft "project." It is the large number of "soft" project, for example, today's campus, badminton, table tennis, rope skipping and so on the grid against the rise of the project, on the one hand is the characteristic of the movement itself determines the project itself.

The change of the mode of production to promote the happening of the gender division of labor, social role change of the underlying factors.

Joan w. Scott to the gender is defined as: "the gender is based on the difference of visible difference of the constituent elements of social relations, is a basic way of said power relations. Gender shows the differences between men and women are not caused by congenital physiological factors, but by human socialization process gradually formed the day after tomorrow, and with the transformation of social production mode and changing. And based on the gender division of labor is on the basis of social gender division of labor. In the primitive society gender division of labor is determined by the people's living environment, social division of labor is the purpose of survival. With the development of productivity, this kind of systematic and social division of labor.

Family, private ownership appeared, the private ownership make family had surplus products, is a symbol of wealth and the remaining products, men create wealth means he is dominant in the family. Women's social status has plummeted. 
Closely related to the mode of production and social division of labor. After the social division of labor gradually evolved into a kind of social mechanism. The result is a man in a rule and the dominant position, the woman belongs to the subordinate and the dominant position. Is the gender division of labor determines the women's social status changes, its performance model, that is, we often say that the "men, tying the" mode "inside, outside". Men and women on the division of labor caused the men have to policy-making and inequality.

\section{Wushu education and relationship between male gender role}

Martial arts in our national culture, extensive and profound, not only has profound significance, and reflects a masculine loftiness everywhere. Every time we recall fearless trounced the strongman Mr. He, for revenge was known as the "sick man of Asia" Chinese national humiliation, when we think of Bruce lee's kung fu at the time of the great influence of the global, martial arts, the Chinese traditional and ancient culture has its strong strength and the huge influence of profound and permanent frames on the history of the clock, until today, its influence still make us proud and excited.

In the animal kingdom, is the nature of their competition, is also the iconic symbol of male and female respectively. People originated in animals, the survival of the pressure forced the man always put yourself in the position of a competition. Both work and life, competition is everywhere. Competition in sports is a typical reflection of male competition.

Energetic for is one of the basic spirit of Chinese martial arts, it embodies is tenacious, indomitable courage, for the fighting spirit of the strong."Yi yue: day line, the gentleman to self-reliance. Of topography, the gentleman to hold world with virtue. This means we have to like a day (natural) strong cheng du jin jian, in worked hard to treat people doing things, forever. At the same time its take measures like the earth, can let all things. We learn by martial arts, also emphasizes the unyielding. Through the process of exercising martial arts to hone the will, perseverance, and courage increases." The shelf plate every day, kung fu, growing", at the same time, wushu practice should pay attention to fine, gas, god, and action of internal and external coordination, system of martial arts study, but the speed of the development of the human body, stamina, agility, flexibility, strength, coordination, etc. In many boxing martial arts pays attention to breathing and intention to cooperate, the internal environment of human body as well as the improvement of each viscera function, physical enhancement can play a role. Stubborn body is self-efficacy reflects male, and masculinity appearance.

\section{Conclusions}

Associated with men's temperament is skilled, motivated, bear, competitiveness, take the initiative, abstract cognition and sense of responsibility, etc. The division of labor is produced with the development of science and technology and the whole society change, this change is the development trend of the whole country and the world, no one can stop. We advocate through the martial arts education to reconstruct the masculinity of the society as a whole, it is not to all the teenagers to learn martial arts, nor encourage and boost patriarchal and restoring the male-dominated system of authority in the past, also not and "women take some rights, but in the current society tightly with the development of The Times makes the pace of a work, life and study, social responsibility is becoming more and more big under this premise, we through the martial arts of the traditional excellent culture of digging, in succession on the basis of the use of its social value, to call brave, resolute, aggressive, magnanimous, decisive, atmosphere and so on these elite masculinity return to nature. Needless to say, when a macho youth, has the good in them long before they can afford the responsibility of the individual, family, country and the society as a whole. That's what we need to cultivate teenagers today masculinity nature real purpose. 


\section{References}

[1]Jiang Chen. Plans to save the boy's revelation. [N]. The dongguan daily, 2013,12,23 (B02)

[2]R.W. Cornell. Masculinity [J]. Beijing: social sciences academic press. 2003:97

[3]Tan Chunxi. "man" education of virtual and real [N]. Weifang daily, 2013,12,6 (006).

[4]Luo Yongyi QiuJun. Based on sports ontology concept of "pleasure" sports teaching reflection [J]. Journal of Beijing sports university, 2015, 20 (2) : 105-111 